\section{Malignant eccrine spiradeno- cylindroma and parotid gland involvement in Brooke Spiegler syndrome}

Giulia Merlo, ${ }^{1}$ Marianna Pesce, ${ }^{2}$ Tiziana Borra, ${ }^{3}$ Ileana Orejuela, ${ }^{2}$ Francesca Pasquali, ${ }^{4}$ Renzo Panizza, ${ }^{2}$ Marco Ghiglione, ${ }^{2}$ Federica Grosso ${ }^{5}$

${ }^{1}$ Dermatology Unit, SS. Antonio e Biagio e Cesare Arrigo Hospital, Alessandria, Italy; ${ }^{2}$ Department of Plastic and Reconstructive Surgery, SS. Antonio e Biagio e Cesare Arrigo Hospital, Alessandria, Italy; ${ }^{3}$ Department of Pathology, SS. Antonio e Biagio e Cesare Arrigo Hospital, Alessandria, Italy; ${ }^{4}$ Vulnology Unit, Local Health Department of Alessandria, Italy; 5Translational Medicine, Integrated Activities Research and Innovation Department (DAIRI), SS. Antonio e Biagio e Cesare Arrigo Hospital, Alessandria, Italy

\begin{abstract}
Brooke-Spiegler syndrome is a rare disorder, characterized by the development of skin adnexal tumors, including cylindromas, trichoepitheliomas, spiradenomas. Although these neoplasms are benign in most patients, a malignant transformation can rarely occur. Furthermore, an occasional association between cutaneous adnexal tumors and basal cell adenoma as well as adenocarcinoma of the parotid gland has been rarely described, with approximately 20 cases reported. We report a case of BSS presenting with a malignant eccrine spiradenocylindroma, in a patient with previous history of parotid basal cell tumor.
\end{abstract}

\section{Introduction}

Brooke-Spiegler syndrome (BSS) is a rare autosomal dominant disorder, characterized by the development of skin adnexal tumors, including cylindromas, trichoepitheliomas, spiradenocylindromas, and spiradenomas. ${ }^{1}$

Although these neoplasms are benign in most patients, a malignant transformation can rarely occur. ${ }^{2}$ Up to now, less than 50 cases of malignant evolution have been reported in literature. . $^{3-5}$

Furthermore, an occasional association between cutaneous adnexal tumors and basal cell adenoma as well as adenocarcinoma of the parotid gland has been rarely described, with approximately 20 cases reported. -10 $^{-10}$

We report a case of BSS presenting with a malignant eccrine spiradenocylindroma, in a patient with previous history of parotid basal cell tumor.

\section{Case report}

A 61-year-old Caucasian man was referred to our hospital with an occipital skin lesion, progressively increasing in size over the last 2 years, without any evidence of previous local trauma. He was diagnosed elsewhere with von Recklinghausen's disease based on the presence of several papules on the face, clinically interpreted as neurofibromas, and on the family history including his father and his daughter with similar lesions on face and scalp. No other comorbidities were reported except a previous surgery for the removal of a parotid basal cell tumor of uncertain malignant potential 5 years before.

At clinical examination, a painful, soft nodular swelling of approximately $3 \times 3 \mathrm{~cm}$ in the occipital area was present (Figure 1A). Neither secretions nor skin changes were detected. The lesion was firm and adherent to the deeper planes. In the surrounding skin, multiple pink, firm, smaller papules of the same appearance could be appreciated. Skin biopsy of the occipital nodule was consistent with a malignant eccrine spiradenocylindroma (Figure 1BD).

On the frontal area many other similar but smaller skin-colored papules with a smooth surface were also observed (Figure 2). On histological examination, these lesions were found to be cylindromas and spiradenomas.

The malignant spiroadenocylindroma was circumferentially excised after marking for surgical excision with $2-\mathrm{cm}$ free-margins. The raw area was covered with a full thickness skin graft taken from the groin and sutured over the raw area. The surgical procedure was performed under local anesthesia and postoperative course was uneventful. Our patient refused to perform the molecular genetic testing for CYLD mutation. However, based on the histological findings and the suggestive family history, the patient was diagnosed with BSS with malignant transformation.

\section{Discussion}

BSS is a rare genetic disorder, resulting from a mutation in the CYLD gene located
Correspondence: Giulia Merlo, Dermatology Unit, Azienda Ospedaliera SS. Antonio e Biagio e Cesare Arrigo, Via Venezia 16, 15121, Alessandria, Italy.

Tel.: +39.0131206492.

E-mail: giulia.merlo@ospedale.al.it

Key words: Dermatology; Genetics; Skin cancer.

Contributions: GM and MP wrote the manuscript; TB was the pathologist who examined skin biopsies; FP, IO, RP and MG operated on the patient; FG contributed to writing and supervised the whole work. All authors read and approved the final version of the manuscript.

Conflict of interest: The authors declare no potential conflict of interest.

Funding: None.

Ethical approval and consent to participate: the patient gave his informed consent to the images being published.

Availability of data and material: Data and materials are available by the authors.

Please cite this article as: Merlo G, Pesce M, Borra T, et al. Malignant eccrine spiradenocylindroma and parotid gland involvement in Brooke Spiegler syndrome. Dermatol Rep 2022;14:9418.

Received for publication: 17 November 2021. Accepted for publication: 4 December 2021.

This work is licensed under a Creative Commons Attribution-NonCommercial 4.0 International License (CC BY-NC 4.0).

${ }^{\circ}$ Copyright: the Author(s), 2022

Licensee PAGEPress, Italy

Dermatology Reports 2022; 14:9418

doi:10.4081/dr.2022.9418

Publisher's note: All claims expressed in this article are solely those of the authors and do not necessarily represent those of their affiliated organizations, or those of the publisher, the editors and the reviewers. Any product that may be evaluated in this article or claim that may be made by its manufacturer is not guaranteed or endorsed by the publisher.

on chromosome 16q12-q13, and marked by the development of skin tumors, including cylindroma, spiradenoma, spiradenocylindroma and trichoepithelioma. ${ }^{11}$ As in our patient, this syndrome typically becomes clinically apparent in adolescence or young adulthood. ${ }^{1}$

It's not always easy to recognize the disease, also due to its rarity. Our patient was previously misdiagnosed as neurofibromatosis. Other similar cases of diagnostic error have been described in the literature, ${ }^{12}$ 
mainly resulting from an inaccurate collection of the medical history or a superficial clinical examination. However, considering the lack of other clinical features typical of neurofibromatosis, the long-time history of multiple adnexal skin neoplasms, the positive family history and the histological find- ings, it was possible to correctly orient the diagnosis.

Although skin neoplasms in BSS are usually benign, very few cases of malignant transformation have been described, ${ }^{2-}$ $5,7,10,13,14$ occurring approximately in 5\% of the patients. ${ }^{12}$ Malign evolution occurs in

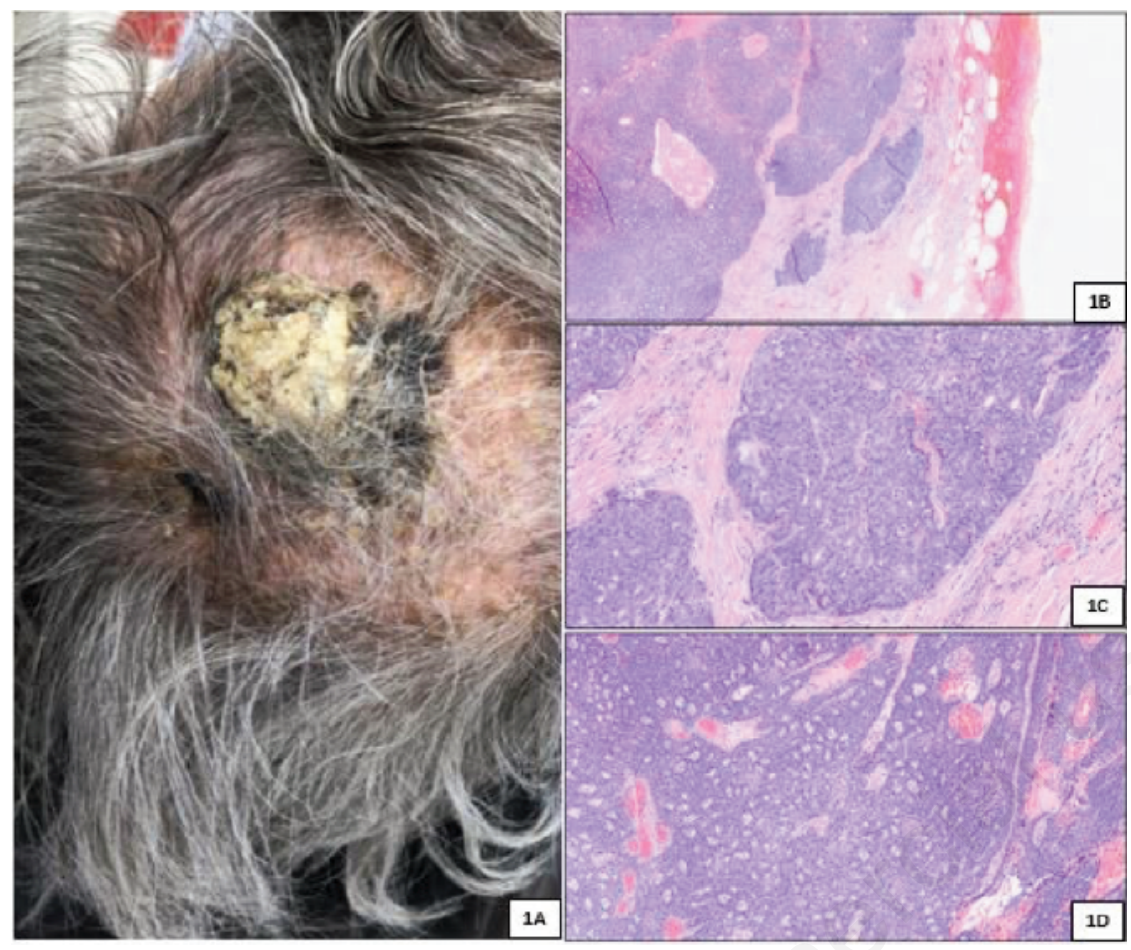

Figure 1. A) Parieto-occipital spiroadenocylindroma. B) Malignant clues include infiltrative growth $(\mathrm{H} \& \mathrm{E}, 4 \mathrm{x}) . \mathrm{C})$ Other features of malignancy are loss of the dual cell population, cytological atypia and increased mitotic activity (H\&E, 20x). D) Histology displays round nodules composed of two types of cells, clear cells and dark cells (H\&E, 10x).

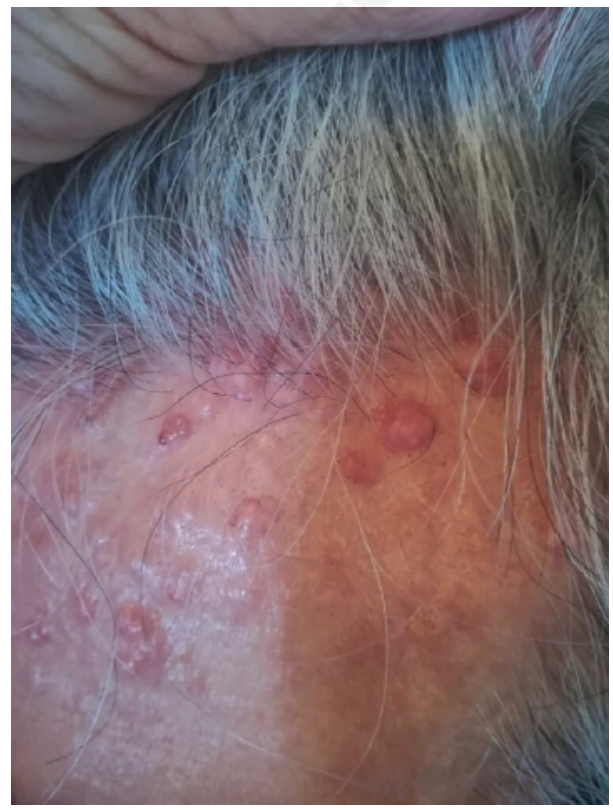

Figure 2. Skin-colored papules on the frontal area. older patients with BSS, ranging from 50 to 80 years old. Scalp seems to be the most commonly affected site. ${ }^{5}$ Rapid growth, ulceration, bleeding and pain should be viewed with suspicion. ${ }^{7,14}$

Moreover, salivary gland involvement is a condition that can be very rarely associated with BSS, with no more than 20 cases reported in the literature..$^{1,6-10,15}$ In his large series on BSS, Kazakov described 2 out of 106 patients who developed salivary gland cancer in addition to skin lesions. ${ }^{1}$ Salivary gland neoplasms are mainly represented by basal cell adenoma as well as adenocarcinoma of the parotid gland, less frequently by submandibular or other minor salivary glands involvement. ${ }^{1,8}$

\section{Conclusions}

Our case represents a very rare example of BSS associated with both parotid involvement and malignant transformation of a skin adnexal tumor. Malignant evolution, although infrequent, should always be excluded through long-term follow-up of BSS patients, to ensure an early treatment and a better prognosis.

\section{References}

1. Kazakov DV. Brooke-Spiegler Syndrome and Phenotypic Variants: An Update. Review Head Neck Pathol 2016;10:125-30.

2. Kazakov DV, Zelger B, Rutten A, et al. Morphologic diversity of malignant neoplasms arising in preexisting spiradenoma, cylindroma and spiradenocylindroma based on the study of 24 cases, sporadic or occurring in the setting of Brooke-Spiegler syndrome. Am J Surg Pathol 2009;33:705-19.

3. Gerretsen AL, van der Putte SC, Deenstra W, et al. Cutaneous cylindroma with malignant transformation. Cancer 1993;72:1618-23.

4. Kuklani RM, Glavin FL, Bhattacharyya I. Malignant cylindroma of the scalp arising in a setting of multiple cylindromatosis: a case report. Head Neck Pathol 2009;3:315-9.

5. Pichler M, Thuile T, Kluge R, et al. Metastatic cylindrocarcinoma in Brooke-Spiegler Syndrome - Report of a case and review of the literature. J Dtsch Dermatol Ges 2021;19:125-8.

6. Malzone MG, Campanile AC, Losito NS, et al. Brooke-Spiegler syndrome presenting multiple concurrent cutaneous and parotid gland neoplasms: 
cytologic findings on fine-needle sample and description of a novel mutation of the CYLD gene. Diagn Cytopathol 2015;43:654-8.

7. Vasenina V, Cibull T, Stadlan N. Brooke-Spiegler Syndrome With Cervical Spine Lesion. Cureus 2020;12:e10982.

8. Kalina P, El-Azhary R. Brooke-spiegler syndrome with multiple scalp cylindromas and bilateral parotid gland adenomas. Case Rep Radiol 2012;2012: 249583.

9. Scott AR, Faquin WC, Deschler DG. Parotid mass in a woman with multiple cutaneous cylindromas. Head Neck 2010;32:684-7.
10. Grossmann P, Vanecek T, Steiner P, et al. Novel and recurrent germline and somatic mutations in a cohort of 67 patients from 48 families with BrookeSpiegler syndrome including the phenotypic variant of multiple familial trichoepitheliomas and correlation with the histopathologic findings in 379 biopsy specimens. Am J Dermatopathol 2013;35:34-44.

11. Blake PW, Toro JR. Update of cylindromatosis gene (CYLD) mutations in Brooke-Spiegler syndrome: novel insights into the role of deubiquitination in cell signaling. Review Hum Mutat 2009;30:1025-36.

12. Denadai R, Stelini RF, Roberto WM, et al. Brooke-Spiegler syndrome clinically misdiagnosed as neurofibromatosis type 1. J Craniofac Surg 2015;26:323-5.

13. Pizinger K, Michal M. Malignant cylindroma in Brooke-Spiegler syndrome. Dermatology 2000;201:255-7.

14. Durani BK, Kurzen H, Jaeckel A, et al. Malignant transformation of multiple dermal cylindromas. $\mathrm{Br} \mathrm{J}$ Dermatol 2001;145:653-6.

15. Köstler E, J Schönlebe, T Mentzel, et al. Psoriasis and Brooke-Spiegler syndrome with multiple malignancies. J Eur Acad Dermatol Venereol 2005;19:380-1. 